'Unidad de Tratamiento del Ataque Cerebrovascular (UTAC), Servicio de Neurología, Departamento de Medicina, Clínica Alemana de Santiago. Facultad de Medicina Clínica Alemana-Universidad del Desarrollo. Santiago, Chile.

Trabajo no recibió financiamiento. Los autores declaran no tener conflictos de interés.

Recibido el 25 de marzo de 2019, aceptado el 13 de agosto de 2019.

Correspondencia a: Dr. Víctor Navia Servicio de Neurología Clínica Alemana de Santiago.

Manquehue Norte 1410, $10^{\circ}$ piso. Vitacura, Santiago, Chile. vnavia@alemana.cl

\section{Trombolisis endovenosa post reversión de acenocumarol con complejo de protrombina. Caso clínico}

\author{
VÍCTOR NAVIA ${ }^{1}$, RENÉ RIVEROS ${ }^{1}$, ALEJANDRO BRUNSER ${ }^{1}$
}

We report an 89-year-old male under oral anticoagulant therapy with a therapeutic international normalized ratio, presenting at the emergency room with right side hemiparesis and aphasia. Neuroimaging was compatible with an acute middle cerebral artery ischemic stroke. Anticoagulation was reverted with the use of four factor prothrombin complex, followed by thrombolysis with alteplase, with a favorable evolution, returning to his basal functional status.

(Rev Med Chile 2019; 147: 932-934)

Key words: Anticoagulants; Prothrombin; Thrombolytic Therapy.

L os antagonistas de la vitamina $\mathrm{K}$ (AVK) se usan ampliamente para prevenir ataques cerebrovasculares isquémicos agudos (ACV) en pacientes portadores de fibrilación auricular (FA). Existe sin embargo, un riesgo anual de ACV cercano al 1,7\% incluso en aquellos con índice normalizado internacional (INR) en rango terapeutico ${ }^{1}$.

De acuerdo a las guías internacionales no se recomienda la trombolisis endovenosa (IVT) para pacientes con INR $>1,7^{2}$.

Los resultados de ensayos animales demuestran un riesgo de sangrado excesivo después de el uso de IVT con un INR elevado ${ }^{3}$.

La anticoagulación inducida por AVK puede revertirse rápidamente mediante la infusión intravenosa de concentrado de complejo de protrombina de 4 factores (4F-PCC) y vitamina K. En un modelo tromboembólico de roedores pretratados con VKA , PCC previno la hemorragia secundaria post-IVT ${ }^{3}$.

En humanos se reportan sólo unos pocos casos de IVT en pacientes con ACV usando PCC para revertir anticoagulación con antagonistas de vitamina $\mathrm{K}^{4,5}$.
Reportamos el caso clínico de un paciente de 89 años usuario de antagonistas de vitamina $\mathrm{k}$ con INR terapéutico que debe revertirse la terapia anticoagulante previo al uso de terapia trombolítica para manejo agudo de su ataque cerebrovascular.

Paciente de 89 años, sexo masculino, diestro con antecedente de fibrilación auricular, enfermedad del nodo, usuario de marcapasos, hipertensión arterial, diabetes tipo 2 no insulino requirente, Guillain Barré con mínima secuela motora crural, usuario de terapia anticoagulante con acenocumarol, amlodipino y metformina, laboralmente activo, es traído por hijo al servicio de urgencia por un cuadro de 13 min de evolución de pérdida súbita de fuerza de la extremidad superior derecha asociada a asimetría facial marcada y afasia de expresión. Ingresa normotenso, con HGT de 134, en Glasgow 14, al examen neurológico destaca agitado, comprende solo algunas órdenes simples, no responde mes ni edad, existe una hemianopsia derecha, una paresia facial central derecha marca$\mathrm{da}$, paresia de la extremidad superior derecha leve, afasia global de predominio expresivo, disartria y extinción táctil. Su escala de NIHSS de ingreso 
es de 11 puntos. La tomografía axial computada (TAC) de cerebro realizada a los 16 min de ingreso no demuestra lesiones hemorrágicas (Figura 1A), la TAC de perfusión cerebral por rapid, que es un software automatizado de evaluación de perfusión por TAC (Figura 1-B) demuestra la ausencia de areas infartadas $(\mathrm{CBF}<30 \%$ de $0 \mathrm{ml})$, pero zonas claras de penumbra u oligemia isquémica (Tmax $>6$ seg de $12 \mathrm{ml}$ ). La angiografía por tomografía axial computada de vasos cervicales y vasos intracraneanos sin oclusiones relevantes.

Dentro de los exámenes de sangre se recibe un INR de 2, que contraindica IVT. Dada la buena funcionalidad previa del paciente y su gran compromiso neurológico, se decide revertir la anticoagulación con complejo de protrombina (octaplex), logrando un INR de 1,2 a los $30 \mathrm{~min}$, se procede posteriormente a realizar IVT a una dosis de $0,9 \mathrm{mg} / \mathrm{kg}$ con un bolo de 7,6 mg seguido de infusión en 1 h de 70, 5 mg. La presión arterial previo al alteplase es de 134/70. La terapía se desarrolla con presión arterial estable y sin eventos adversos terminando con un déficit neurológico leve mínimo caracterizado por; asimetría facial y disatria lo que puntúa un NIHSS de 2 puntos.

El paciente es ingresado a la unidad de tratamiento de ataque cerebrovascular para manejo, monitorización y estudio.

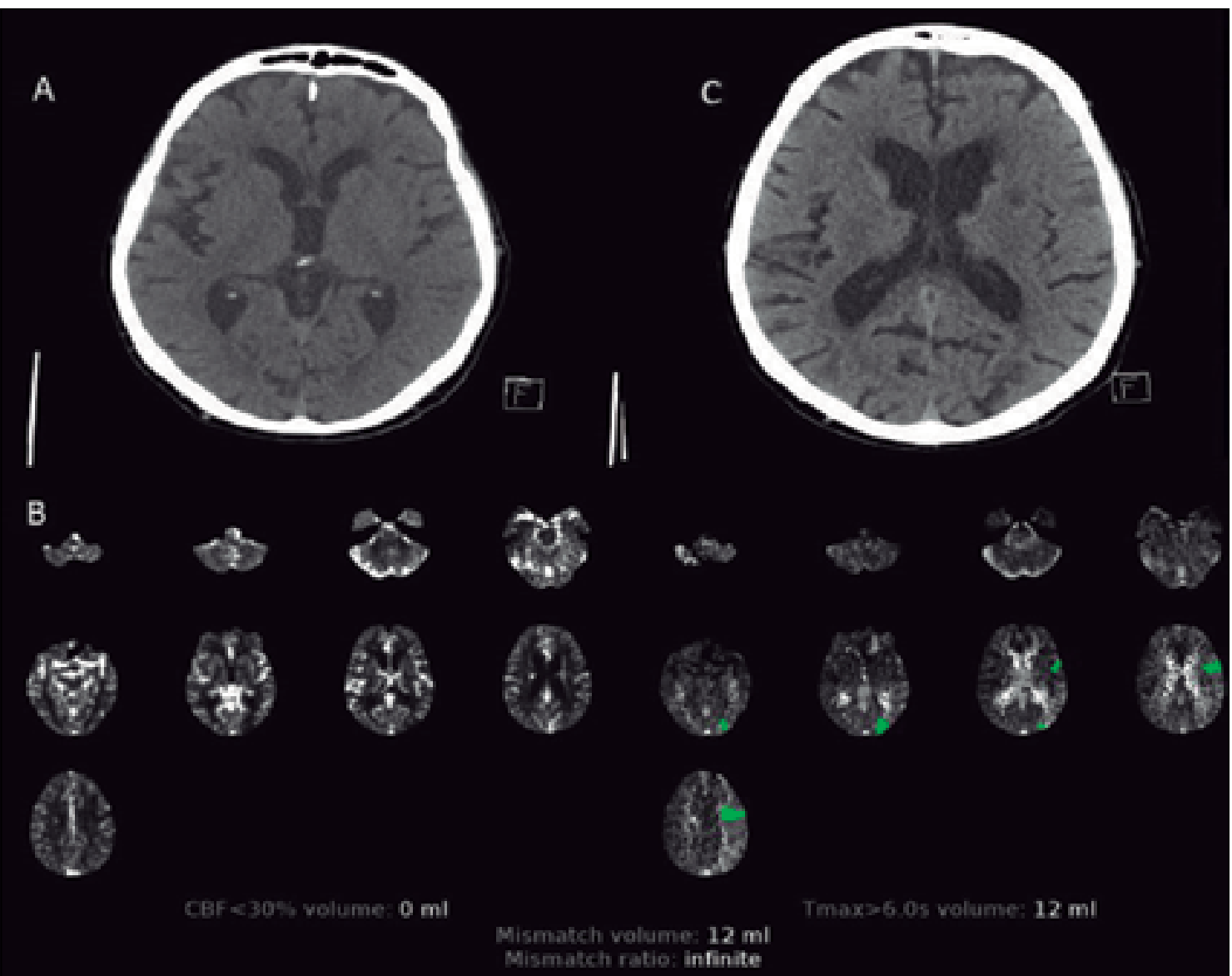

RAPID

Figura 1. La tomografía axial computada (TAC) de cerebro realizada a los 16 min de ingreso no demuestra lesiones hemorrágicas (A), el test de rapid (B) demuestra la ausencia de areas infartadas (CBF $<30 \%$ de $0 \mathrm{ml}$ ), pero zonas claras de penumbra u oligemia isquemica (Tmax $>6$ seg de $12 \mathrm{ml}$ ). TAC de cerebro de control a las $24 \mathrm{~h}$ (C): Infarto agudo frontal precentral izquierdo, sin evidencia de transformación hemorrágica. 
A las $24 \mathrm{~h}$ destaca fluencia de lenguaje levemente disminuida con trastorno de la nominación de algunos objetos. Resto de examen neurológico normal. NIHSS: 1 punto.

TAC de cerebro de control a las $24 \mathrm{~h}$ (Figura 1C): Infarto agudo frontal precentral izquierdo, sin evidencia de transformación hemorrágica.

A los 4 días el paciente es dado de alta en su condición basal, se cambia el acenocumarol por dabigatrán $110 \mathrm{mg}$ cada $12 \mathrm{~h}$ y se agrega estatinas a dosis elevadas a su terapia de base.

\section{Discusión}

La terapia de reperfusión tiene una fuerte evidencia para su uso en ACV, favoreciendo la funcionalidad de los pacientes con un NNT de $7^{6}$.

La IVT con alteplase es un tratamiento efectivo para la disolución del trombo en $\mathrm{ACV}$, aunque conlleva un riesgo de hemorragia intracraneal sintomática que va de $5 \%$ al $7 \%$ en ciertas condiciones $^{5,7,8}$.

La tasa de complicaciones trombóticas posterior a la reversión con $4 \mathrm{~F}-\mathrm{PCC}$ de hemorragia en usuarios de AVK es de 2,5\% a los 30 días en un reciente meta-análisis 9 .

Existe evidencia de que los pacientes con ACV tratados con VKA y que presentan INR $>1,7$ podrían beneficiarse de la IVT después de la reversión de la anticoagulación ${ }^{5,10}$ pero se requiere confirmación por ensayos controlados aleatorios bien diseñados, nuestro caso clínico está en línea con esta hipotesis.

La publicación de este reporte cuenta con la autorización explícita del paciente.

\section{Referencias}

1. Prabhakaran S, Rivolta J, Vieira JR, Rincon F, Stillman J, Marshal RS, et al. Symptomatic intracerebral hemorrhage among eligible warfarin-treated patients receiving intravenous tissue plasminogen activator for acute ischemic stroke. Arch Neurol 2010; 67: 559-63.

2. Powers WJ, Rabinstein AA, Ackerson T, Adeoye OM, Bambakidis NC, Becker K, et al; American Heart Association Stroke Council. 2018 guidelines for the early management of patients with acute ischemic stroke: a guideline for healthcare professionals from the American Heart Association/American Stroke Association. Stroke 2018; 49: e46-e110.

3. Sun L, Zhou W, Ploen R, Heiland S, Zorn M, Veltkamp R. Rapid reversal of anticoagulation prevents excessive secondary hemorrhage after thrombolysis in a thromboembolic model in rats. Stroke 2011; 42: 3524-9.

4. Jalini S, Jin AY, Taylor SW. Reversal of warfarin anticoagulation wit prothrombin complex concentrate before thrombolysis for acute stroke Cerebrovascular Dis 2012; 33: 597.

5. Chausson N, Soumah D, Aghasaryan M, Altarcha T. Reversal of Vitamin K Antagonist Therapy Before Thrombolysis for Acute Ischemic Stroke, Stroke 2018; 49: 2526-8.

6. Joung-Ho Rha and Jeffrey L. The Impact of Recanalization on Ischemic Stroke Outcome A Meta-Analysis Stroke 2007; 38: 967-73.

7. Sandercock P, Wardlaw JM, Lindley RI, et al. The benefits and harms of intravenous thrombolysis with recombinant tissue plasminogen activator within $6 \mathrm{~h}$ of acute ischemic stroke (the third international stroke trial [IST-3]): a randomized controlled trial. Lancet 2012; 379: 2352-63.

8. Lees KR, Bluhmki E, von Kummer R, et al. Time to treatment with intravenous alteplase and outcome in stroke: an updated pooled analysis of ECASS, ATLANTIS, NINDS, and EPITHET trials. Lancet 2010; 375: 1695-703.

9. Brekelmans MPA, Ginkel KV, Daams JG, Hutten BA, Middeldorp S, Coppens M. Bene ts and harms of 4-factor prothrombin complex concentrate for reversal of vitamin $\mathrm{K}$ antagonist associated bleeding: a systematic review and meta-analysis. J Thromb Thrombolysis. 2017; 44: 118-29.

10. Rebello LC, Haussen DC, Belagaje S, Anderson A, Frankel M, Nogueira RG. Endovascular treatment for acute ischemic stroke in the setting of anticoagulation. Stroke 2015; 46: 3536-9. 